\title{
Need for a Prescriptive Taxonomy of Interaction for Mathematical Cognitive Tools
}

\author{
Kamran Sedig \\ Cognitive Engineering Laboratory \\ The University of Western Ontario \\ London, Ontario, Canada \\ sedig@uwo.ca
}

\begin{abstract}
Cognitive tools encode and display information in different representational forms. Through interaction with these representations, people can make sense of and reason about the information. Mathematical cognitive tools can allow people to interact with mathematical ideas in ways and levels not hitherto possible. These tools can support reasoning and enhance the experience and understanding of mathematics. However, currently, there exists no prescriptive taxonomy of interaction with mathematical representations to guide designers of mathematical cognitive tools. Given different representations, cognitive activities, and users, such a taxonomy can help designers know what interaction techniques to use, when, and to what end. This paper discusses the need for the development of a comprehensive prescriptive taxonomy of interaction with visual representations used in mathematical cognitive tools. Additionally, the paper outlines some inter-related lines of action that can be followed to develop such a taxonomy.
\end{abstract}

\section{Introduction and Background}

Mathematical knowledge is an important asset in knowledge societies, where people work with their minds more than their bodies. Yet, due to the highly formal nature of mathematics, its abstract representational forms, and the means by which it is communicated, many people do not find mathematical ideas readily understandable, nor engaging. New computational technologies can help. Just as technological tools enhanced the physical reach and capabilities of people during the Industrial Revolution and helped increase their productivity, computational technologies can enhance our minds [1]. As Norman [2] states, "Without external aids, memory, thought, and reasoning are all constrained... The real powers come from devising external aids that enhance cognitive abilities... It is [external aids] that make us smart."

One category of external aids are cognitive tools or technologies, tools that people can use to support, guide, and enhance their mental activities and increase their productivity. ${ }^{1}$ These tools allow for the encoding and displaying of information in differ

\footnotetext{
1 Many terms have been used to signify cognitive tools: mindtools, instruments and technologies of the mind, information artifacts, mental gadgets, and so on [1, 2, 3]. This paper uses the term cognitive tools to refer to interactive computational devices, specifically interactive software applications.
} 
ent representational forms. Through interaction with these representations, different cognitive tasks can be performed, such as visualizing, analyzing, interpreting, meaning making, decision making, planning, problem solving, and learning [4]. These tools can be our partners in cognition, carrying some of the cognitive load and helping us perform mental activities of different degrees of complexity, some even impossible to accomplish without their aid [3,4]. Examples of these tools include interactive visualization software to customize and observe climatological patterns, mind mapping tools to help externalize and organize thoughts and concepts, and online interactive mathematical applets to investigate how velocity and position graphs relate. Mathematical cognitive tools (MCTs) are a subset of cognitive tools, intended to support interaction with mathematical objects, concepts, and problems. By using appropriate, innovative human-computer interaction techniques, MCTs can allow people to explore mathematical ideas in ways and levels not hitherto possible, supporting reasoning and enhancing understanding of mathematics, and, hence, contributing to the creation of a more mathematically literate and productive society.

Two of the main components of MCTs are: representations and interaction.

\subsection{Representations}

External representations play a central role in the communication and understanding of mathematics. There are two broad types of representations: algebraic and visual. The former are made of formal, language-like symbols; the latter are made of imagebased, graphical notations [5]. This paper is concerned with the latter: visual mathematical representations (VMRs). Research shows that human beings process and manage visual representations more efficiently than algebraic representations $[5,6]$.

Some examples of VMRs from different areas of mathematics include 3D and 4D geometric structures, statistical graphs, and tiling patterns. These graphical notations encode structural, relational, functional, and semantic properties of concepts, objects, patterns, problems, and ideas in mathematics. In other words, they embody the meaning that the images try to communicate. Effectiveness and appropriateness of an image depend to a large degree on how its users interpret it and make sense of its inherent meaning as well as how well it fits and supports the cognitive task $[2,5,7]$. Making sense of and reasoning with VMRs often depends on a process in which the user browses a visual image, poses 'what if' questions to it, and rearranges it until its meaning is gradually understood and then uses the representation for performing cognitive tasks [8]. This cognitive process of decoding and application can be mediated and supported through appropriate user interaction with VMRs.

\subsection{Interaction}

Interaction with a VMR has two aspects: a user acting upon the VMR through the intermediary of a human-computer interface, and the VMR communicating back through some form of reaction or response [9]. This addition of reaction, responsiveness, or interactivity to static visual representations enhances their communicative power and leads to some general benefits [7, 10, 11, 12]: supporting emergent understanding of encoded ideas; making mental manipulation of ideas easier; providing opportunities for experimentation, discovery, and hypothetical reasoning; supporting 
'doing' rather than 'watching'; facilitating acquisition of qualitative insight into the nature of representations; supporting cognitive tracing and computational offloading by allowing users to think in partnership with a representation; and, serving as a coordinator between the internal mental models of users and an external representation.

Although, as discussed above, we know that interaction has general benefits, designing interaction to effectively mediate, support and enhance cognitive activities is challenging $[9,10]$. It has been shown that using the wrong type of interaction can actually constrain human thinking and have negative, undesirable consequences [10, 13]. Just as users use different reasoning and thinking styles to approach different cognitive tasks [4], so they need different interaction techniques to mediate and support their reasoning with different VMRs [14]. Interaction technique and style play a crucial role in how users' minds are engaged with VMRs - that is, how they reason, what cognitive strategies they employ, how deeply they process the visual information, what knowledge they construct, and how well they learn $[9,13,14,15]$. The types of VMRs used and the various given cognitive tasks for an MCT have a significant bearing on the types of interaction that are effective and should be used. Just as the effectiveness of a representation depends on whether it is appropriate for a given task, a given interaction technique should also fit the task, as it extends and mediates the representation in the process of cognitive activities.

\subsection{Design Taxonomies}

Being a young research area, much of the early research in cognitive technologies has been devoted to the building of domain-specific tools. This process of tool building has been needed for experimentation and has led to dozens of innovative interaction design techniques across different types of cognitive tools. When designing interaction for VMR-based MCTs, however, there exists no conceptual framework to guide the design. Often times, designers use techniques developed to support productivity tasks, which may not fit learning tasks. These tasks have different requirements and goals. In productivity tools, the goal is to reduce the user's cognitive effort; whereas in learning tasks, the goal is to engage users in mindful cognition $[9,15]$. To address this type of problem in human-computer interaction, task and interface taxonomies are often developed [10]. A taxonomy abstracts, articulates, decomposes, and organizes a problem space, which, consequently, can facilitate analysis and understanding, provide opportunities for comparing different techniques, guide designers, and foster innovation and advancement [10]. There are two broad types of taxonomies: descriptive and prescriptive. Descriptive taxonomies characterize the decomposed elements of the problem space and provide examples of them. Prescriptive taxonomies, on the other hand, prescribe design rules and guidelines for the elements of the problem space and can provide best-practice examples of existing systems and techniques. Currently, there exist a number of taxonomies dealing with visualization and visual representations, interface styles, interactive externalizations, and interface tasks [3,10,12,16,17]. Though some of the terms and ideas used in these taxonomies are relevant to the objectives of designing MCTs, none of them prescriptively address how these tools should be designed to fit and support the cognitive tasks of the users. 
Recently, Sedig and Sumner [9] have developed a descriptive taxonomy of interaction with VMRs. This taxonomy organizes and characterizes the different interaction techniques that can be used in MCTs. The techniques are organized and characterized according to their common features. The taxonomy consists of three primary interactions (conversing, manipulating, and navigating) and twelve secondary interactions (animating, annotating, chunking, constructing, cutting, filtering, fragmenting, probing, rearranging, repicturing, scoping, and searching). The taxonomy also identifies ten dimensions of interactivity (affordance, computational offloading, constraints, control, distance, feedback, flow, focus, participation, and scaffolding). Though a step in the right direction, this taxonomy is not prescriptive.

This paper proposes that there is a need for systematic research to develop a comprehensive prescriptive taxonomy of interaction for VMR-based MCTs. As online MCTs are now being delivered on the WWW as applets [17], and with the pervasiveness of the Internet, MCTs can virtually affect millions of people in terms of their application of mathematics in knowledge development and production. Since interaction is one of the central, distinguishing features of e-learning, a prescriptive taxonomy of interaction can guide the design and evaluation of not only MCTs, but also other types of cognitive tools. Mathematics has a rich repertoire of representational forms. As such, many interface concepts and interaction techniques developed for MCTs can transfer to cognitive tools embedding other knowledge domains.

\section{A Proposed Strategy}

This section proposes a strategy for conducting research to develop a prescriptive taxonomy of interaction and outlines some of the methodological actions needed to achieve the objectives of this type of research.

\subsection{Problem Statement}

Before discussing how a prescriptive taxonomy can be developed and what lines of action are needed for this research, this paper presents a problem statement to demonstrate some questions that may arise in the course of designing interaction for MCTs. The problem statement can be as follows:

Given a VMR, a cognitive task or activity, and a prototypical user, what interaction technique(s) should be designed to render an MCT effectivethat is, what technique(s) would support and enhance the user who is engaged with the cognitive task involving the VMR? How should interactivity (e.g., feedback or scaffolding) be operationalized to enhance the technique(s)?

Three scenarios are presented to situate the problem in real cases:

1) Chemists need to have good cognitive maps of the structures of different $3 \mathrm{D}$ lattices in order to reason about crystals. Given a tetrahedral lattice structure, the goal is to help chemistry students form an accurate cognitive map of the structure so they can mentally visualize it and reason about similar structures. What technique should be used to support the task of forming a cognitive map of a 3D 
structure? Should the user 'walk' on the structure or 'fly' through and/or around it? Should the user annotate the structure by marking it with visual breadcrumbs?

2) Four-dimensional polytopes can have many and varied geometric components, making their visualized image complex, cluttered, and noisy. Given a rendered visualization of a 4D polytope, a mathematician must make sense of the image by identifying, locating, and distinguishing its faces and cells, which are some typical cognitive tasks performed on visualizations [16]. What interaction techniques support these cognitive tasks? Should the user interact with the VMR directly or not? Should the visualization be fragmentable?

3) Polyhedra solids can be transformed from one shape to another by truncating and/or augmenting their vertices and edges. Given a set of polyhedra solids, the goal is to explore and investigate how these transformations take place. Should these transformation processes be animated or should the user manipulate these VMRs to morph them? Should the interaction be continuous or discrete? Should the user interact with these VMRs directly or indirectly? What are the tradeoffs?

Without a framework within which to analyze and answer these questions, it is difficult to know how to design an MCT, the design remains arbitrary, and it is not easy to predict the impact the design may have on its users.

\subsection{Towards a Prescriptive Taxonomy of Interaction}

To address the types of questions posed in the above problem statement, we need to develop a prescriptive taxonomy of interaction with VMRs. Such a taxonomy should contain methods, rules, and guidelines for the design of MCTs in order to facilitate and support performance of given cognitive tasks by some prototypical users. These rules and guidelines should help with the analysis, prediction, and prescription of aspects of design as they pertain to mind-MCT interaction. The taxonomy should be tied to appropriate cognitive frameworks and provide a vocabulary that the designers can use when investigating and evaluating the implications of their design choices.

In order to develop such a taxonomy, several inter-related lines of action are proposed below. Although these lines of action are presented sequentially, aspects of them can overlap and take place simultaneously.

1) Creation of test-bed MCTs. In order for the taxonomy to cover a wide range of VMRs and cognitive tasks, it is necessary to have a varied and rich set of MCTs which instantiate different techniques and can be used as test beds. Our research group has already created several MCTs dealing with areas such as 2D transformation geometry, 2D and 3D lattices, 2D polygonal patterns, 3D solids, 4D complex polytope visualizations, state-transition diagrams, and visual mathematical programming. We are in the process of building more tools. However, what is needed is for research groups to collaborate to build more tools and share them with each other. We need a large repertoire of these test-bed MCTs.

2) Designing and conducting of usability studies. Interaction techniques used in the MCTs should be tested with quantitative and qualitative methods to assess their effectiveness and observe their patterns of use. Conducting such studies will allow for comparison of different techniques. As more studies are conducted, features of these techniques can be validated, abstracted, and generalized $[1,10]$. This process will also help create profiles for prototypical users. There have been 
a few such studies, and the results clearly suggest that interaction techniques affect learning for better or worse. There needs to be a more concerted and systematic way of conducting these studies. A descriptive taxonomy of interaction, such as the one developed by Sedig and Sumner [9], can provide a framework within which these studies are conducted.

3) Development of rules and guidelines. Results obtained from (2) should be analyzed to distill rules and guidelines for interaction with VMRs. Some important issues to consider will be: type of rules (e.g., if-then vs. procedural decision trees); representation of rules; and, level of granularity and degree of abstractness of rules. There are a few studies whose findings can be regarded as general, embryonic rules [13, 14, 15]. For instance, from one of the studies [13], the following rule can be distilled: if a VMR represents a concept (e.g., rotation), and the concept is made of sub-concepts encoded as different visual elements in the VMR (e.g., angle of rotation and center of rotation), and the cognitive task is to understand the concept, then gradual removal of the VMR's visual elements helps users take notice of its sub-concepts. Such rules and guidelines, however, should be further developed and validated to generalize to a wide set of user types and VMRs. This can be done by incorporating and evaluating a rule in the context of other MCTs so that the rule is refined and its validity increases.

4) Organization of rules into taxonomy. A three dimensional structure, such as a cube, can be used to help organize and onto which map the rules developed in (3). The axes of the cube would represent the three-tuple (user, cognitive task, VMR), and its cells would point to a set of rules prescribing which interaction techniques to use and would provide examples of best-practice MCTs that incorporate these techniques. For instance, an instantiation of the rule in (3) is embodied in an MCT that a designer can study as a practical application of that rule [5].

5) Decomposition of taxonomy axes. There exist classification schemes for visual representations [18], cognitive tasks [19, 20], and user types [19]. For instance, a structural classification of six types of visual representations along 10 characteristics has been developed [18], though not explicitly about VMRs. This and other classification schemes can be used as starting points for decomposing the taxonomy axes. Through evaluation and analysis, these schemes can gradually evolve and be refined. The strategy to be used for decomposing the axes in (4) can play an important role in the usability and effectiveness of the proposed taxonomy [10]. Issues to consider include type and level of granularity of decomposition. For instance, should decomposition be in terms of higher-level, conceptual tasks such as 'formation of cognitive maps', or should it be in terms of lower-level tasks such as 'locating an element', or should it be hierarchical, encompassing several levels? Furthermore, should the VMR axis be decomposed using broad categories or specific categories? For instance, the axis may have specific elements such as 3D lattices and 3D solids, or a broader category such as 3D structures.

6) Validation of rules and taxonomy. Validation of the rules and the taxonomy will be an important line of action. By necessity, the development and validation of a prescriptive taxonomy is evolutionary. However, there are some general steps that can increase the validity of such taxonomies [1]: 1) repeated evaluation of rules using reliable testing instruments, 2) designing and evaluating MCTs with and without the use of the taxonomy and comparing the results, and 3) having outside experts review, evaluate, and test the rules and the categories. 


\section{Summary}

Interaction plays an important role in helping learners (or users) make sense of visual mathematical representations. Given different visual mathematical representations, cognitive tasks, and users, designers often need to use different interaction techniques. Therefore, they need to know what interaction techniques to use, when, and to what end. Using the wrong type of interaction can constrain human thinking and have negative, undesirable consequences for learners. Human-computer interaction designers use design taxonomies, particularly prescriptive ones, to facilitate analysis and understanding of design requirements, to provide opportunities for comparing different techniques, and to guide designers. Currently, there exist no design taxonomies to guide the design of interactive mathematical cognitive tools containing visual representations. This paper calls for the development of a comprehensive prescriptive taxonomy of interaction for mathematical cognitive tools.

To develop such a taxonomy, the paper proposes several inter-related lines of action. These lines of action are as follows: creating a large repertoire of mathematical cognitive tools that can be used as test-beds for experimenting with different design requirements; designing and conducting usability studies to investigate and compare different interaction techniques to find out how they support understanding of different mathematical concepts and to what end; developing general rules and guidelines for interaction design as a result of usability studies performed with a large set of test-bed tools; taxonomizing design rules and pointing designers to tools that embody best-practice examples of these prescriptive rules; and, as the rules and the taxonomy evolve, constantly evaluating them to increase their validity.

The scope of such a research agenda calls for collaboration among researchers in this area. As mathematics has a very rich repertoire of representational forms, many of the guidelines and interaction techniques developed in this area can transfer to other cognitive tools embedding other knowledge domains.

\section{References}

1. Jonassen, DH (ed.): Handbook of research for educational communications and technology. Simon \& Schuster Macmillan (1996)

2. Norman, DA: Things that make us smart: Defining human attributes in the age of the machine. Addison Wesley (1993)

3. Beynon, B, Nehaniv, CL, Dautenhahn, K (eds.): Cognitive technology: Instruments of mind. Proceedings of International Cognitive Technology Conference. Springer-Verlag (2001)

4. Lajoie, S (ed.): Computers as cognitive tools. Lawrence Erlbaum Assoc. (2000)

5. Skemp, RR: The psychology of learning mathematics. Penguin Books (1986)

6. Card, SK, Mackinlay, JD, Shneiderman, B. (eds.): Readings in information visualization: Using vision to think. Morgan Kaufmann Publishers (1999)

7. Strothotte, T: Computational visualization: Graphics, abstraction, and interactivity. Springer-Verlag (1998)

8. Stylianou, D: On the interaction of visualization and analysis: The negotiation of a visual representation in expert problem solving. Journal of Mathematical Behavior 21: (2002) 303-317 
9. Sedig, K, Sumner, M: Characterizing interaction with visual mathematical representations. International Journal of Computers for Mathematical Learning. Kluwer Academic Publishers (Under review)

10. Carroll, JM (ed.): Designing interaction: Psychology at the human-computer interface. Cambridge University Press (1991)

11. Hanson, A, Munzner, T, Francis, G: Interactive methods for visualizable geometry. IEEE Computer, (1994) 27(7): 73-83

12. Tweedie, LA: Characterizing interactive externalizations. In Proceedings of CHI '97, (1997) 375-382

13. Sedig, K, Klawe, M, Westrom, M: Role of interface manipulation style and scaffolding on cognition and concept learning in learnware. ACM Transactions on Computer-Human Interaction, (2001) 1(8), 34-59

14. Sedig, K, Rowhani, S, Morey, J, Liang, H: Application of information visualization techniques to the design of a mathematical mindtool: A usability study. Palgrave Macmillan Ltd. Information Visualization, (2003) 2(3): 142-160

15. Golightly, D: Harnessing the interface for domain learning. In Proceedings of CHI '96, (1996) 37-38

16. Keller PR, Keller MM: Visual cues: Practical data visualization. IEEE Computer Society (1993)

17. Gadanidis, G, Sedig, K, Liang, H: Designing online mathematical investigations. Journal of Computers in Mathematics and Science Teaching. Association for the Advancement of Computing in Education (In press)

18. Lohse, GL, Biolsi, K, Walker, N, Rueter, HH: A classification of visual representations. Communications of the ACM, (1994) 37(12): 36-49

19. Jonassen, DH, Tessmer, M \& Hannum, WH: Task analysis methods for instructional design. Lawrence Erlbaum Associates (1999)

20. Schraagen, MC, Chipman, SF \& Shalin, VL (eds.): Cognitive task analysis. Lawrence Erlbaum Assoc (2000) 